\title{
SIRT1 Deacetylates Tau and Reduces Pathogenic Tau Spread in a Mouse Model of Tauopathy
}

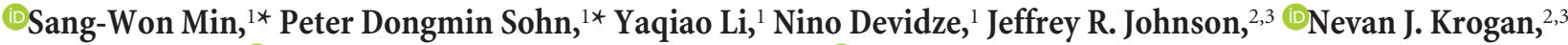 \\ Eliezer Masliah, ${ }^{\circ}$ Sie-Ann Mok, ${ }^{4}$ Jason E. Gestwicki, ${ }^{5}$ and ${ }^{\mathbb{D}}$ Li Gan ${ }^{1,4}$ \\ ${ }^{1}$ Gladstone Institute of Neurological Disease, ${ }^{2}$ Gladstone Institute of Cardiovascular Disease, ${ }^{3}$ Department of Cellular and Molecular Pharmacology, \\ ${ }^{4}$ Department of Neurology, ${ }^{5}$ Institute for Neurodegenerative Disease, Department of Pharmaceutical Chemistry, University of California, San Francisco, San \\ Francisco, California 94158, and 'Department of Neuroscience, University of California, San Diego, La Jolla, California 92093
}

Hyperacetylation of tau has been implicated in neurodegeneration and cognitive decline in tauopathy brains. The nicotinamide adenosine dinucleotide-dependent class-III protein deacetylase SIRT1 is one of the major enzymes involved in removal of acetyl groups from tau in vitro. However, whether SIRT1 regulates acetylation of pathogenic tau and ameliorates tau-mediated pathogenesis remains unclear. Here, we report deacetylating activity of SIRT1 for acetylated Lys174 (K174) of tau in tauP301S transgenic mice with a brainspecific SIRT1 deletion. We show that SIRT1 deficiency leads to exacerbation of premature mortality, synapse loss, and behavioral disinhibition in tauP301S transgenic mice of both sexes. By contrast, SIRT1 overexpression by stereotaxic delivery of adeno-associated virus that encodes SIRT1 into the hippocampus reduces acetylated K174 tau. Furthermore, SIRT1 overexpression significantly attenuates the spread of tau pathology into anatomically connected brain regions of tauP301S transgenic mice of both sexes. These findings suggest the functional importance of SIRT1 in regulating pathogenic tau acetylation and in suppressing the spread of tau pathology in vivo.

Key words: acetylation; dementia; SIRT1; tau; tau spread

Significance Statement

In neurodegenerative disorders with inclusions of microtubule-associated protein tau, aberrant lysine acetylation of tau plays critical roles in promoting tau accumulation and toxicity. Identifying strategies to deacetylate tau could interfere with disease progression; however, little is known about how pathogenic tau is deacetylated in vivo. Here we show that the protein deacetylase SIRT1 reduces tau acetylation in a mouse model of neurodegeneration. SIRT1 deficiency in the brain aggravates synapse loss and behavioral disinhibition, and SIRT1 overexpression ameliorates propagation of tau pathology.

\section{Introduction}

Tauopathies, including Alzheimer's disease (AD) and frontotemporal dementia and parkinsonism linked to chromosome 17 (FTDP-17), are a class of neurodegenerative disorders associated with inclusions of microtubule-associated protein tau. Tau

\footnotetext{
Received Aug. 21, 2017; revised Feb. 2, 2018; accepted March 1, 2018

Author contributions: S.-W.M., P.D.S., and L.G. designed research; S.-W.M., P.D.S., Y.L., N.D., J.R.J., E.M., and S.-A.M. performed research; N.D., N.J.K., E.M., and J.E.G. contributed unpublished reagents/analytic tools; S.-W.M., P.D.S., Y.L., J.R.J., and L.G. analyzed data; S.-W.M., P.D.S., and L.G. wrote the paper.

This work was supported by the National Institutes of Health (1R01AG054214-01A1, U54NS100717, R01AG051390; to L.G.) and the Tau Consortium (to L.G.). Behavioral data were obtained with the help of the Gladstone Institutes' Neurobehavioral Core (supported by National Institutes of Health Grant P30NS065780). The Gladstone Institutes received support from National Center for Research Resources Grant RR18928. We thank Dr. Leonard Petrucelli (Mayo Clinic) for AAV-SIRT1, Dr. Peter Davies (Feinstein Institute) for the MC1 antibody, and Latrice Gross and Erica Nguyen for administrative assistance.

*S.-W.M. and P.D.S. contributed equally to this work.

The authors declare no competing financial interests.

Correspondence should be addressed to Dr. Li Gan, Gladstone Institutes of Neurological Disease, 1650 Owens Street, San Francisco, CA 94158, E-mail: Igan@gladstone.ucsf.edu.

DOI:10.1523/JNEUROSCI.2369-17.2018

Copyright $@ 2018$ the authors $\quad 0270-6474 / 18 / 383680-09 \$ 15.00 / 0$
}

is extensively modified post-translationally, and abnormal post-translational modifications are associated with tau pathology (Morris et al., 2015; Wang and Mandelkow, 2016). We and others reported that lysine acetylation of tau is markedly elevated in human tauopathy brains (Min et al., 2010, 2015; Cohen et al., 2011; Grinberg et al., 2013; Irwin et al., 2013; Sohn et al., 2016; Tracy et al., 2016). Depending on the specific lysine acetylated, the acetylation of tau affects different aspects of tau biology, including tau turnover (Min et al., 2010, 2015), synaptic plasticity (Tracy et al., 2016), tau mis-sorting (Sohn et al., 2016), aggregation (Cohen et al., 2011), and phosphorylation (Cook et al., 2014). Acetylation at Lys174 (K174) has been shown to play a crucial role in tau homeostasis and cognitive deficits in a mouse model of tauopathy (Min et al., 2015).

SIRT1 is a nicotinamide adenosine dinucleotide-dependent class-III protein deacetylase and has neuroprotective functions (Gan and Mucke, 2008; Min et al., 2013; Ng et al., 2015). In human tauopathy brains, SIRT1 levels are reduced and negatively correlate with the amount of tau accumulation (Julien et al., 2009), whereas acetylated K174 tau accumulates in the early stages of hu- 
man tauopathy brains (Min et al., 2015), suggesting a possible pathogenic role of SIRT1 deficiency in enhanced K174 tau acetylation and concomitant tau accumulation. Unlike histone deacetylase 6 (HDAC6), which can deacetylate lysine residues within the KXGS motifs in the microtubule-binding domain of tau (Cook et al., 2014), SIRT1 deacetylates lysine residues in the flanking region that encompasses K174 (Min et al., 2010). However, whether SIRT1 deacetylates tau in tauopathy mouse models remains to be elucidated.

Distribution of tau pathology in the CNS has been used for staging progression of AD (Braak and Braak, 1991). Accumulating evidence suggests that cell-to-cell transmission of pathological tau accounts for progressive spread of pathology in tauopathy brains (Clavaguera et al., 2009; Iba et al., 2013; Brettschneider et al., 2015). SIRT1 activation or elevation ameliorates pathology and neurodegeneration in AD (Qin et al., 2006; Kim et al., 2007). Loss of SIRT1 induces impairment of learning and memory (Gao et al., 2010; Michán et al., 2010). Despite the beneficial roles of SIRT1 in neurodegenerative diseases, whether SIRT1 can be protective against tau-mediated pathogenesis and the spread of tau pathology is largely unknown.

In the current study, we used quantitative proteomics to determine whether SIRT1 modulates tau acetylation in vitro. Using a transgenic mouse model of tauopathy with brain-specific SIRT1 deletion, we examined whether SIRT1 deacetylates tau in vivo. To investigate the deleterious effects of SIRT1 deficiency on tau-mediated pathogenesis, we examined premature lethality, synapse loss, and cognitive decline in the tauopathy mouse model with SIRT1 deletion. Finally, using stereotactic intracerebral injection of adenoassociated virus (AAV), we assessed whether SIRT1 overexpression attenuates the spread of tau pathology to different brain regions.

\section{Materials and Methods}

\section{Primary antibodies}

AC312 for ac-K174 tau (Min et al., 2015), MAB359 for ac-K274 tau (Grinberg et al., 2013), Tau-5 (Bio-Source), Sirt-1 (Abcam), GAPDH (Sigma-Aldrich), tubulin (Sigma-Aldrich), PSD-95 (Cell Signaling Technology), GluA-1 (Cell Signaling Technology), synaptophysin (Cell Signaling Technology), and hemagglutinin (HA; Cell Signaling Technology) primary antibodies were purchased. $\mathrm{MCl}$ antibody is a gift from $\mathrm{Dr}$. Peter Davies (Feinstein Institute).

\section{Western blots}

Cells or mouse brain tissues were homogenized in RIPA buffer containing protease inhibitor mixture (Sigma-Aldrich), $1 \mathrm{~mm}$ phenylmethyl sulfonyl fluoride, phosphatase inhibitor mixture (Sigma-Aldrich), $5 \mathrm{~mm}$ nicotinamide (Sigma-Aldrich), and $1 \mu \mathrm{m}$ trichostatin A (Sigma-Aldrich). After sonication, lysates from mouse brain were centrifuged at 20,000 $\times$ $g$ at $4^{\circ} \mathrm{C}$ for $20 \mathrm{~min}$. Supernatants were collected, and protein concentrations were determined by the Bradford assay (Bio-Rad). The same amounts of proteins were resolved on a $4-12 \%$ SDS-PAGE gel (Invitrogen), transferred to nitrocellulose membrane (GE Healthcare), and probed with appropriate antibodies. Immunoblots were visualized by enhanced chemiluminescence (Pierce) and quantified by densitometry and ImageJ software (National Institutes of Health).

\section{Stable isotope-labeled amino acids in cell culture}

HEK293 cells were maintained with either light or heavy medium for $>10$ passages. Ten $10 \mathrm{~cm}$ plates, which were either cultured in light or heavy medium, were transfected with $5 \mu \mathrm{g}$ of C-terminal flag-tagged human tau plasmids along with p300 and SIRT1 plasmids. After $48 \mathrm{~h}$ of incubation from transfection, cells were detached and lysed with RIPA buffer (50 mu Tris, pH 7.5, 1 mm EDTA, 150 mm NaCl, 0.5\% NP-40) with protease inhibitor mixtures (Sigma-Aldrich). Protein concentration was measured using Bradford assay (Bio-Rad), and the same amounts of protein lysates were immunoprecipitated with flag-M2 agarose beads (Sigma-Aldrich), resolved by SDS-PAGE, and visualized with Coomassie staining. Bands corresponding to the molecular weight of tau were excised from the gel and subjected to in-gel trypsin digestion (Rosenfeld et al., 1992; Hellman et al., 1995). Gel bands were diced and evaporated to dryness. The gel pieces were then rehydrated with trypsin buffer. The digestion was incubated overnight at $37^{\circ} \mathrm{C}$. Samples were desalted with C18 ZipTips (Millipore) using the manufacturer's recommended proto$\mathrm{col}$ and evaporated to dryness. The samples were resuspended in $0.1 \%$ formic acid for mass spectrometric analysis.

\section{Mass spectrometry}

For stable isotope-labeled amino acids in cell culture (SILAC) analyses, "light" and "heavy" tau digests were combined equally, based on densitometric analysis of the intensity of the Coomassie-stained bands. The samples were analyzed by an LTQ Orbitrap Elite mass spectrometry system, equipped with an easy nLC 1000 liquid chromatography system (Thermo Fisher Scientific). Two microliters of each sample were injected onto a $75 \mu \mathrm{m} \times 25 \mathrm{~cm}$ fritted capillary column (New Objective) packed with $1.9 \mu \mathrm{m}$ ReproSil-Pur C18 AQ particles. The column sprayed directly into the mass spectrometer via a NanoSpray Flex nanoelectrospray. Samples were separated by a 115 min gradient from 5 to $30 \%$ B $(100 \%$ acetonitrile, $0.1 \%$ formic acid) followed by a $5 \mathrm{~min}$ wash at $100 \% \mathrm{~B}$. The mass spectrometer operated in data-dependent mode collected one survey scan in the Orbitrap at $120,000 \times$ resolution (FWHM). Raw mass spectrometry data were analyzed by the MaxQuant algorithm (version 1.3.0.5; Cox and Mann, 2008). The sequence database used was SwissProt Human. Variable modifications allowed were lysine acetylation, methionine oxidation, and N-terminal acetylation. Lys 6 was indicated for heavy SILAC labeling. All other parameters used were MaxQuant default parameters. The resulting SILAC ratios were log-transformed and ratios were averaged for peptides detected multiple times in the same sample. The total tau log ratio was calculated by averaging the log ratios of all tau peptides that were either unmodified or that contained oxidized methionine. The total tau log ratio was then subtracted from the log ratio of each acetylated peptide to correct for bias created when light and heavy samples were combined at ratios approximated by gel densitometry.

\section{Mouse breeding}

Mice were housed in a pathogen-free barrier facility with a $12 \mathrm{~h}$ light/dark cycle and ad libitum access to food and water. All animal procedures were performed under guidelines approved by the Institutional Animal Care and Use Committee of the University of California, San Francisco. TauP301S mice were crossed with SIRT1 F/F mice to generate tauP301S; SIRT1 F/+ mice, which were further crossed with SIRT1 F/F mice to generate tauP301S; SIRT1 F/F. To remove SIRT1 activity from neural and glial progenitor cells, SIRT1 F/F mice were crossed with mice expressing Cre recombinase under the control of the Nestin promoter. This breeding generated Nestin-Cre; SIRT1 F/+ mice, which were further crossed with SIRT1 F/F mice to generate Nestin-Cre; SIRT1 F/F mice. Crossing of tauP301S; SIRT1 F/F mice with Nestin-Cre; SIRT1 F/F mice generated four genotypes: SIRT1 F/F, tauP301S; SIRT1 F/F, Nestin-Cre; SIRT1 F/F, and tauP301S; Nestin-Cre; SIRT1 F/F. Gender-balanced groups were used for all experiments.

\section{Postsynaptic density fractionation}

Mice were perfused, and dissected forebrains were homogenized with buffer 2 ( $5 \mathrm{~mm}$ Tris-Cl, $320 \mathrm{~mm}$ sucrose, $\mathrm{pH}$ 7.4), followed by centrifugation at $3600 \times g$ for $10 \mathrm{~min}$ at $4^{\circ} \mathrm{C}$. Pooled supernatants were loaded on Percoll (Sigma-Aldrich) gradient $(23,10$, and $3 \%)$ and centrifuged at $31,400 \times g$ for $7 \mathrm{~min}$ at $4^{\circ} \mathrm{C}$. Fractions that appeared milky were collected and centrifuged at 20,000 $\times g$ for $35 \mathrm{~min}$ at $4^{\circ} \mathrm{C}$ to collect fractions enriched in synaptosomes. Pelleted synaptosomes were further resuspended in buffer 2 with buffer 3 ( 5 mм Tris-Cl, $1 \%$ Triton X-100, pH 7.4) and mixed for 15 min at $4^{\circ} \mathrm{C}$, followed by centrifugation at $32,800 \times g$ for 20 min at $4^{\circ} \mathrm{C}$ to collect the postsynaptic density (PSD)-enriched fraction in a pellet.

\section{Immunohistochemistry and image analysis}

Mice were transcardially perfused with $0.9 \%$ saline. The mouse brain was fixed in $4 \%$ paraformaldehyde for $48 \mathrm{~h}$ and then in $30 \%$ sucrose for $48 \mathrm{~h}$ at $4^{\circ} \mathrm{C}$. A freezing microtome (Leica) was used to cut $30-\mu \mathrm{m}$-thick coronal brain sections. For MC1 and anti-flag immunostaining, sections 
were permeabilized in blocking solution (TBS containing 10\% normal goat serum and $0.3 \%$ Triton X-100) at room temperature for $1 \mathrm{~h}$. Next, sections were incubated in blocking solution containing MC1 (gift from Dr. Peter Davies' laboratory, Feinstein Institute) or flag (Sigma-Aldrich) antibody overnight at $4^{\circ} \mathrm{C}$, followed by incubation with the appropriate Alexa Fluor-conjugated secondary antibodies (Life Technologies). For AC312 (anti-ac-K174 tau) immunostaining, the AC312 antibody was preabsorbed by tau-knock-out mouse brain powder before use to reduce nonspecific binding of the antibody. Antigen retrieval was performed by heating the sections in PBS containing $10 \mathrm{~mm}$ citric acid for $5 \mathrm{~min}$ in a pressure cooker (Cuisinart). Sections were then permeabilized and incubated with blocking solution containing AC312 antibody (1:500; Min et al., 2015) overnight at $4^{\circ} \mathrm{C}$, followed by a $1 \mathrm{~h}$ incubation with peroxidaseconjugated secondary antibody (EMD Millipore) at room temperature. Immunoreactivity was visualized using 3,3' -diaminobenzidine-peroxidase solution (Vector Laboratories) for $1 \mathrm{~min}$ at room temperature. Immunostaining for synaptophysin using SY38 antibody (Millipore) was performed as described previously (Rockenstein et al., 2015). Images were acquired on a Keyence BZ-X7000 microscope with a 10× objective lens and quantified using ImageJ software (National Institutes of Health). Experimenters quantifying immunoreactivity were blind to the mouse genotype and treatment conditions.

\section{Gallyas silver staining}

Frozen brain sections were incubated with $5 \%$ periodic acid, followed by incubation of alkali silver iodide solution. After soaking with $0.5 \%$ of acetic acid solution, the sections were developed in development solution containing ammonium/silver nitrate and tungstosilic acid. Then they were again soaked with $0.5 \%$ of acetic acid solution, before incubation with $0.1 \%$ gold chloride. Finally, they were dehydrated and coverslips with xylol were applied for visualization.

Tau purification, fibrillization, and fluorescent protein labeling Myc-tagged repeat domain (K18) of P301L tau was expressed in Terrific broth media containing sodium chloride $(500 \mathrm{~mm})$ and a small-molecule chaperone, betaine $(10 \mathrm{~mm})$, to improve expression and minimize degradation. Expression was induced with $200 \mu \mathrm{M}$ isopropyl-thio- $\beta$-Dgalactoside for $3.5 \mathrm{~h}$ at $30^{\circ} \mathrm{C}$. Tau was purified as described previously (Barghorn et al., 2005). Purified tau was dialyzed overnight at $4^{\circ} \mathrm{C}$ into aggregation assay buffer (Dulbecco's PBS, pH 7.4, $2 \mathrm{mM} \mathrm{MgCl}_{2}, 1 \mathrm{~mm}$ DTT). Aggregation of tau $(20 \mu \mathrm{M})$ was induced by the addition of a freshly prepared heparin sodium salt solution (Santa Cruz Biotechnology) at a final concentration of $88 \mu \mathrm{g} / \mathrm{ml}$. Tau fibril samples were labeled with Alexa Fluor 647 NHS ester (Invitrogen) at 1:40 molar ratio of dye/tau monomer for $1 \mathrm{~h}$ at room temperature. Samples were centrifuged at $100,000 \times g$ for $1 \mathrm{~h}$ at $4^{\circ} \mathrm{C}$ to remove unreacted free dye. Pellets containing tau fibrils were resuspended in Dulbecco's PBS, pH 7.4, and $2 \mathrm{~mm}$ $\mathrm{MgCl}_{2}$. Tau fibril concentrations were quantified by SDS-PAGE with a set of tau monomer standards.

\section{Stereotaxic injections}

Mice were anesthetized with $2 \%$ isoflurane by inhalation for the duration of surgery, and secured on a stereotaxic frame (Kopf Instruments). Twomonth-old tauP301S transgenic mice were injected stereotaxically with equal amounts $\left(1 \times 10^{13}\right.$ genomic particles $)$ of AAV1 expressing either SIRT1 with flag tag (gift from Dr. Leonard Petrucelli, Mayo Clinic) or GFP control (ViroTek). These injections were injected into the dentate gyrus of the left hippocampus. Meanwhile, $3 \mu \mathrm{l}$ of synthetic tau fibrils with four repeats (K18) were injected into the CA1 region of the right hippocampus. All injections were given at a rate of $0.5 \mu \mathrm{l} / \mathrm{min}$. The following coordinates were used as measured from bregma: for the dentate gyrus, $-2.1 \mathrm{~mm}$ anterior-posterior, $-1.7 \mathrm{~mm}$ medial-lateral, -2.1 $\mathrm{mm}$ dorsal-ventral; for the CA1, $-2.5 \mathrm{~mm}$ anterior-posterior, $+2.0 \mathrm{~mm}$ medial-lateral, $-1.8 \mathrm{~mm}$ dorsal-ventral.

\section{Behavioral tests}

Experimenters were blind to the genotypes or treatments of the mice for all behavioral analyses.

Elevated plus maze. The elevated plus maze (EPM) consisted of two open and two closed arms elevated $60 \mathrm{~cm}$ above the ground. Mice were allowed to habituate in the testing room under dim light for $1 \mathrm{~h}$ before testing. During testing, mice were placed at the junction between the open and closed arms of the plus maze and allowed to explore for $5 \mathrm{~min}$. The maze was cleaned with $70 \%$ alcohol after testing of each mouse. Total distance traveled and time spent in both the open and closed arms were calculated based on infrared photobeam breaks.

Small Y-maze. Mice were placed in the center of a small Y-maze (arm length, $15 \mathrm{~cm}$ ) and spontaneous alternation was recorded in a single continuous $6 \mathrm{~min}$ trial by a live observer. Each of the three arms was designated a letter A, B, and C, and entries into the arms were recorded. The percentage of spontaneous alternation was calculated over the total number of entries possible. The apparatus was cleaned with $70 \%$ ethanol between trials.

Morris water maze. The water maze consisted of a pool $(122 \mathrm{~cm}$ in diameter) containing opaque water $\left(20 \pm 1^{\circ} \mathrm{C}\right)$ and a platform $(14 \mathrm{~cm}$ in diameter) submerged $1.5 \mathrm{~cm}$ below the surface. Hidden platform training (days 1-5) consisted of two sessions (two per day, $2 \mathrm{~h}$ apart), each with three trials. The mouse was placed into the pool at alternating drop locations for each trial. A trial ended when the mouse located the platform. The maximum trial time was $60 \mathrm{~s}$ per trial. Mice that failed to find the platform within the $60 \mathrm{~s}$ trial were led to it and placed on it for $15 \mathrm{~s}$. For probe trial, $48 \mathrm{~h}$ after the final hidden training, mice were returned to the pool with a drop location that was $180^{\circ}$ opposite of original target platform location in the absence of the hidden platform. Performance was measured with an EthoVision video-tracking system (Noldus Information Technology). Visible platform training, where the platform was cued with a mounted black-and-white striped mast, was conducted for four trials after completion of probe trials. The pre-established exclusion criterion was that mice that floated or did not swim would be excluded from analysis.

\section{Experimental design and statistical analysis}

For Western blot of acetylated tau (ac-tau) in the brain lysates and PSD fractions (see Fig. $2 A-D$ ), data were analyzed from six (two males and four females) tauP301S; SIRT1 F/F and seven (four males and three females) Nestin-cre; tauP301S; SIRT1 F/F mice 7-9 months old. For mouse survival measurement (see Fig. $3 A$ ), data were analyzed from both sexes of 19 SIRT1 F/F, 18 Nestin-cre; SIRT1 F/F, 18 tauP301S; SIRT1 F/F, and 20 Nestin-cre; tauP301S; SIRT1 F/F mice. For synaptophysin immunostaining (see Fig. $3 \mathrm{~B}, \mathrm{C}$ ), data were analyzed from six (three males and three females) tauP301S; SIRT1 F/F and six male Nestin-cre; tauP301S; SIRT1 F/F mice 7-9 months old. For Gallyas silver staining (see Fig. 3D), data were analyzed from six (two males and four females) tauP301S; SIRT1 F/F and five male Nestin-cre; tauP301S; SIRT1 F/F mice 7-9 months old. For behavioral tests (see Fig. $4 A-D$ ), data were analyzed from 5-6.5-month-old mice. For the EPM test (see Fig. 4A), data were analyzed from 15 (eight males and seven females) SIRT1 F/F, 15 (11 males and four females) Nestin-cre; SIRT1 F/F, 14 (five males and nine females) tauP301S; SIRT1 F/F, and 10 (seven males and three females) Nestin-cre; tauP301S; SIRT1 F/F mice. For Y-maze and Morris water maze tests (see Fig. $4 B-D$ ), data were analyzed from 11 (eight males and three females) SIRT1 F/F, 11 (seven males and four females) Nestin-cre; SIRT1 F/F, 11 (five males and six females) tauP301S; SIRT1 F/F, and 10 (seven males and three females) Nestin-cre; tauP301S; SIRT1 F/F mice. The same cohort of mice was used for three behavioral tests, and there was loss of a few mice between behavioral tests due to premature mortality of mice with P301S tau expression.

For immunostaining of pathological tau after AAV-SIRT1 injection (see Fig. 5C-F), 2-month-old tauP301S mice were stereotaxically injected with AAV-SIRT1 and tau fibrils and killed 1 month after the injection. AC312 immunostaining (see Fig. 5C,D) was analyzed from 10 (four males and six females) tauP301S mice with AAV-GFP injection and 11 (five males and six females) tauP301 mice with AAV-SIRT1 injection. MC1 immunostaining (see Fig. $5 E, F$ ) was analyzed from 35 to 38 brain sections of 10 (four males and six females) tauP301S mice with AAV-GFP injection and 11 (five males and six females) tauP301 mice with AAVSIRT1 injection.

The Student's $t$ test, one-way ANOVA with Tukey's or Dunnett's post hoc test, and log rank test were performed with Prism 7 (GraphPad). Multilevel mixed effects linear regression model was performed on STATA 12 
A

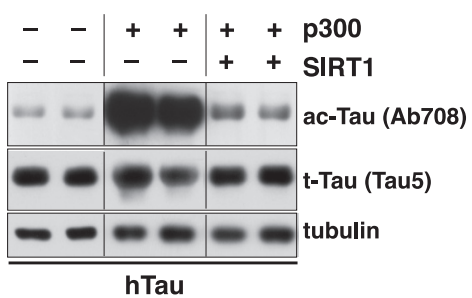

B

\begin{tabular}{|c|c|c|c|c|}
\hline Peptide Sequence & Site & $\begin{array}{l}\text { Log Ratio } \\
\text { Ctrl:Ctrl }\end{array}$ & 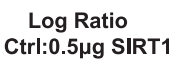 & $\begin{array}{c}\text { Log Ratio } \\
\text { Ctrl:1 } \mu \mathrm{g} \text { SIRT1 }\end{array}$ \\
\hline GAAPPGQK(Acetyl)GQANATR & 163 & -0.085 & -INF & - INF \\
\hline IPAK(Acetyl)TPPAPK & 174 & -0.056 & $-I N F$ & $-I N F$ \\
\hline HQPGGGK(Acetyl)VQIINK & 274 & -0.134 & $-I N F$ & -INF \\
\hline VQIINK(Acetyl)K & 280 & -0.06 & - INF & $-I N F$ \\
\hline IGSLDNITHVPGGGNK(Acetyl)K & 369 & -0.036 & -1.468 & -INF \\
\hline
\end{tabular}

Figure 1. SIRT1 reduces the levels of tau acetylation in vitro. A, Overexpression of SIRT1 significantly reduced p300-mediated tau acetylation in vitro. HEK293T cells were transfected with human 2N4R tau plasmid alone or cotransfected with p300 and/or SIRT1 plasmids. Western blot analysis was performed with a pan-ac-tau antibody (Ab708), pan-t-tau antibody (tau5), and tubulin antibody as a loading control. Representative images from three independent experiments. $\boldsymbol{B}$, The SILAC system revealed downregulation of tau acetylation in multiple lysine sites. Mass spectrometry analyses identified a series of lysine sites of tau whose acetylation was decreased by overexpressing SIRT1. Ctrl:Ctrl compared heavy medium with expression of $h$ Tau, $\mathrm{p} 300$, and control vectors (Ctrl) with light medium with expression of hTau, p300, and control vectors (Ctrl). Ctrl:0.5 $\mu \mathrm{g}$ of SIRT1 compared heavy medium with expression of hTau, p300, and control vectors (Ctrl) with light medium with expression of hTau, p300, and $0.5 \mu \mathrm{g}$ SIRT1 vectors ( $0.5 \mu \mathrm{g} \mathrm{SIRT1).} \mathrm{Ctrl:1.0} \mu \mathrm{g}$ of SIRT1 compared heavy medium with expression of hTau, p300, and control vectors (Ctrl) with light medium with expression of hTau, p300, and $1.0 \mu \mathrm{g}$ SIRT1 vectors (1.0 $\mu \mathrm{g}$ SIRT1). Log ratio was based on the peak of light medium normalized to the peak of heavy medium. - INF, Minus infinity log value due to no peak detection in light medium with SIRT1 expression.

A

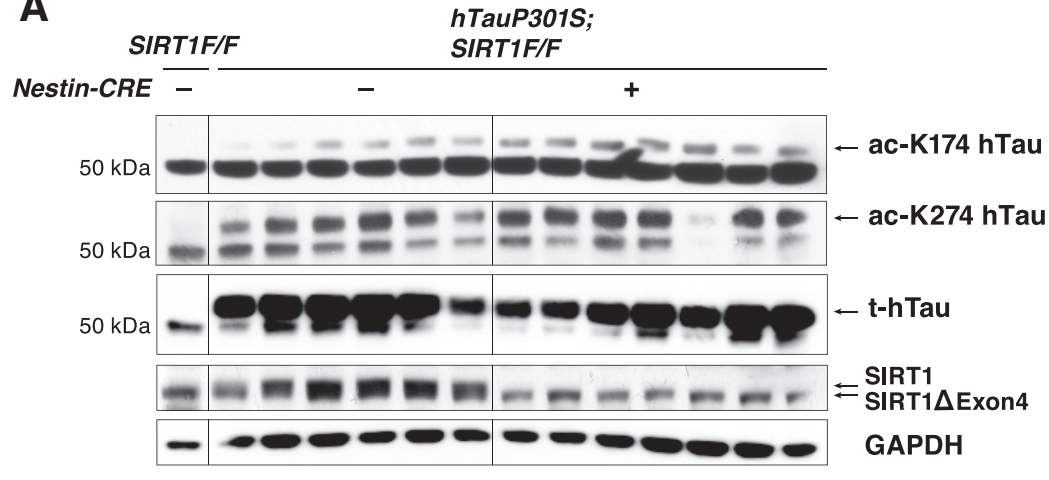

Total Cortical Lysates

C

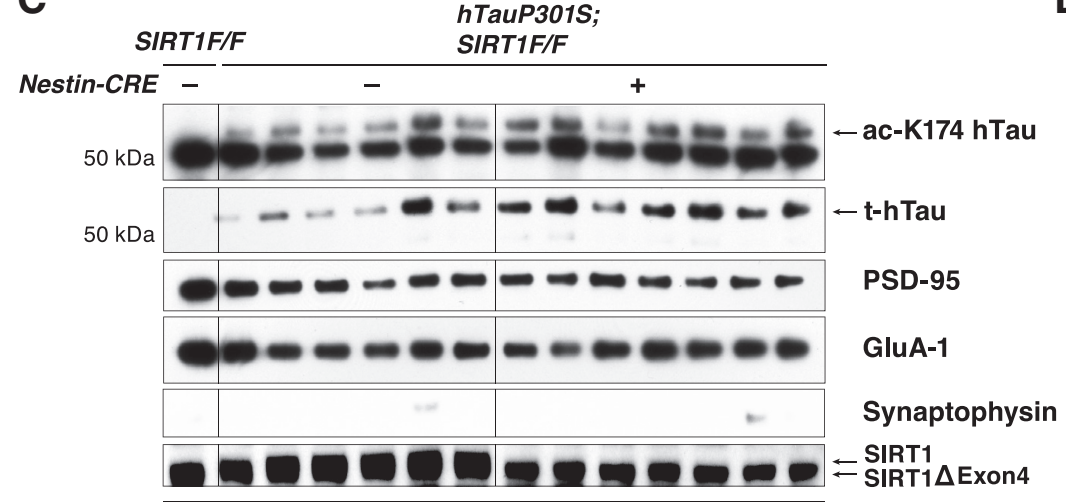

B

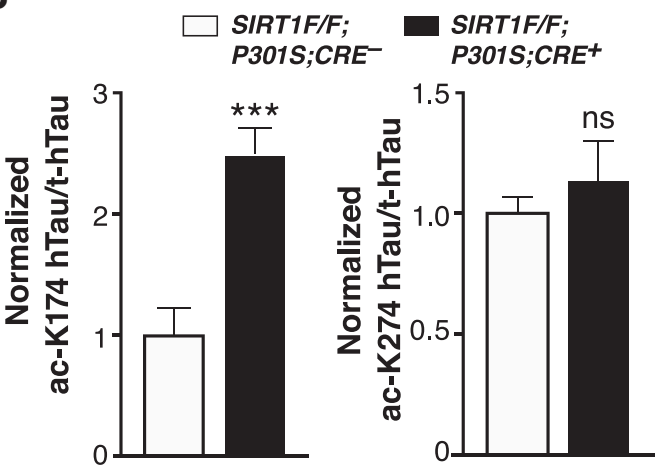

D
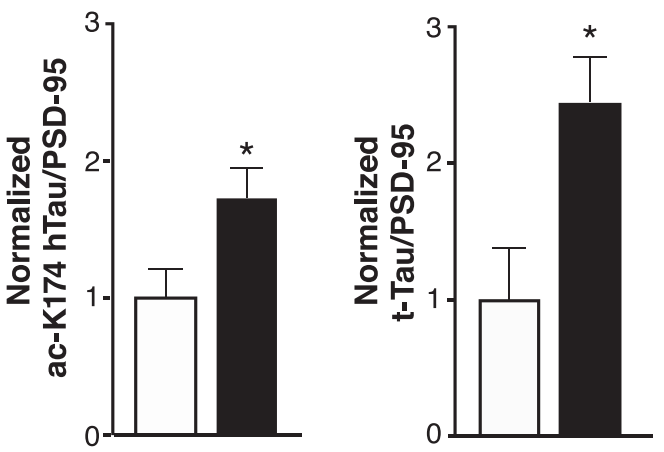

PSD-enriched Fractions

Figure 2. Brain-specific deletion of SIRT1 in vivo selectively increases the levels of ac-K174 tau. $A, B$, Western blot of cortical lysates showed that deleting SIRT1 significantly increased the levels of ac-K174 tau, but not ac-K274 tau. A, Representative Western blot images from cortical lysates. Human tau was identified based on its distinct size of molecular weight that was larger than mouse tau. GAPDH was used as a loading control. B, Quantification showed a significant increase of human ac-K174 tau (AC312), but not human ac-K274 tau (MAB359) by SIRT1 deletion. $n=6-7$ mice per genotype, ${ }^{* * *} p<0.001$, unpaired Student's $t$ test. C, $\boldsymbol{D}$, Western blot of PSD-enriched fractions showed that SIRT1 deletion increased the levels of ac-K174 tau as well as total tau. C, Representative Western blot images from PSD-enriched fractions. Robust expression of PSD-95 and GluA-1 along with the absence of synaptophysin expression indicated the enrichment of PSD fractions. D, Quantification of Western blot showed a significant increase of human ac-K174 tau (AC312) as well as human total tau (Tau5) in PSD-enriched fractions by SIRT1 deletion. $n=6-7$ mice per genotype, ${ }^{*} p<0.05$, unpaired Student's $t$ test. Values are means \pm SEM.

(StataCorp) software for statistical comparisons. Outliers are pre-established as data outside of mean $\pm 2 \mathrm{SD}$. The level of significance was set as $p<0.05$.

\section{Results}

SIRT1 induces nondiscriminative deacetylation of tau in vitro We reported that SIRT1 deacetylates tau in vitro (Min et al., 2010). Consistent with this finding, we confirmed that while p300 overexpression induced ac-tau, SIRT1 deacetylated tau in HEK293 cells using pan-ac-tau antibody Ab708 (Fig. 1A). To identify which lysine sites are preferentially deacetylated by SIRT1 in vitro, the SILAC method was performed to quantify SIRT1-mediated deacetylation in HEK293 cells. HEK293 cells were grown in either heavy-isotopelabeled medium or light-isotope-labeled medium. After cells were passaged for $\geq 10$ times, HEK293 cells in heavy medium were transfected with tau and p300, and HEK293 cells in light 
A

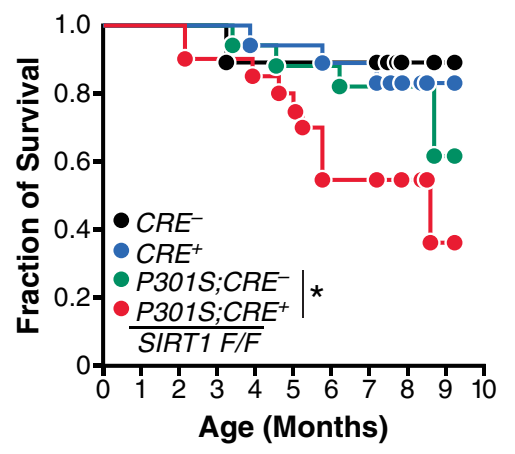

B

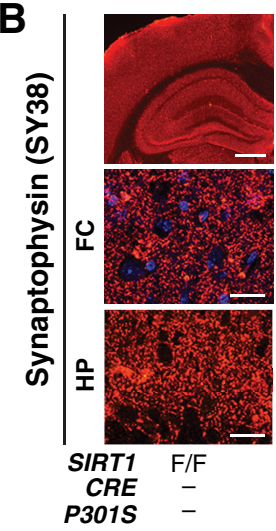

D
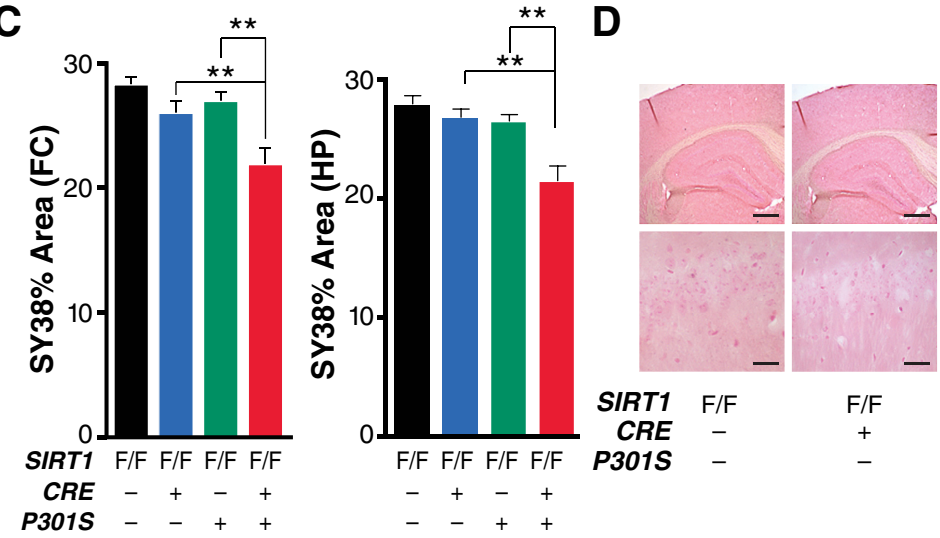
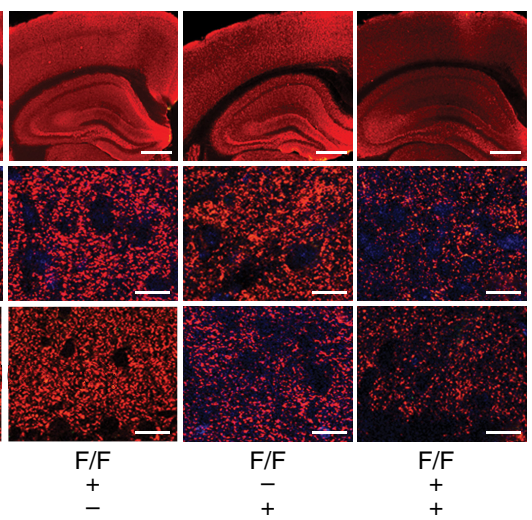

Figure 3. SIRT1 deletion exacerbates premature lethality and synapse loss in tauP301S mice. A, Kaplan-Meier survival analysis showed a significant reduction of survival rate in tauP301S mice

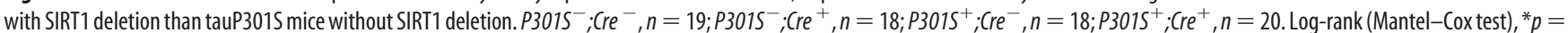
$0.0329\left(\right.$ P3015 $^{+} ; \mathrm{Cre}^{-}$vs P3015 ${ }^{+}$; $\left(\mathrm{re}^{+}\right) \cdot \mathbf{B}, \mathbf{C}$, Synapse loss was exacerbated in tauP3015 mice with SIRT1 deletion. B, Synaptophysin (SY38) immunostaining in both the frontal cortex (FC) and hippocampus (HP). Scale bars: first row, $250 \mu \mathrm{m}$; second and third rows, $25 \mu \mathrm{m}$. C, Quantification of percentage of SY38-positive area showed a significant reduction of synapses in tauP3015 mice with SIRT1 deletion. $n=6$ mice per genotype, ${ }^{* *} p<0.01$, one-way ANOVA, Tukey's post hoctest. $D$, Formation of neurofibrillary tangle (NFT) was not altered in tauP301S mice with SIRT1 deletion. Gallyas silver staining was performed to investigate the degree of NFT in either tauP301S mice or tauP301S mice with SIRT1 deletion. No NFT was identified in tauP301S-negative mice as expected. $n=5-6$ mice per genotype. Scale bars: first row, $250 \mu \mathrm{m}$; second row, $25 \mu \mathrm{m}$. Values are means \pm SEM.

medium were transfected with tau, p300, and SIRT1. Using the SILAC method, we identified five lysines acetylated by p300 expression (i.e., K163, K174, K274, K280, and K369; Fig. 1B). All five lysines were deacetylated by SIRT1 expression (Fig. 1B). Thus, SIRT1-induced deacetylation of tau does not appear to exhibit site preference when overexpressed in cultured cells.

\section{Brain-specific SIRT1 deletion elevates levels of tau acetylation} on K174 in tauP301S mice

To test whether SIRT1 regulates tau acetylation in vivo, SIRT1 was deleted in the brains of tauP301S mice. Expression of Nestincre resulted in deletion of exon 4 of SIRT1, which then led to inactivation of SIRT1 in the brain (Fig. 2A). Levels of total human tau or mouse tau were not significantly affected by SIRT1 deletion. Levels of human ac-K174 tau detected with AC312 antibody were significantly elevated by loss of SIRT1 $\left(t_{(11)}=4.69, p=0.0007\right.$, unpaired $t$ test), whereas levels of ac-K274 tau remained unaffected (Fig. 2A,B). Thus, unlike an in vitro study where SIRT1 deacetylates multiple acetyllysines indiscriminately (Min et al., 2010), endogenous SIRT1 appears to preferentially remove an acetyl group from ac-K174 tau in tauP301S mouse brains.

Tau is an axonal protein; however, tau is often mislocalized to the postsynaptic compartment, especially under pathological conditions. To determine whether ac-tau is mislocalized in the postsynaptic dendritic compartment, we used Percoll gradient centrifugation to isolate PSD-enriched fraction characterized by the presence of PSD-95 and the absence of the presynaptic protein synaptophysin (Fig. 2C). SIRT1 deletion led to a significant increase of human acK174 tau in the PSD-enriched fractions $\left(t_{(11)}=2.47, p=0.0312\right.$, unpaired $t$ test; Fig. $2 C, D$ ). Interestingly, levels of total human tau in the PSD-enriched fraction were also significantly increased by SIRT 1 deletion $\left(t_{(11)}=2.77, p=0.0183\right.$, unpaired $t$ test; Fig. $\left.2 C, D\right)$.

\section{SIRT1 deletion exacerbated synaptic loss and led to} behavioral deficits in tauP301S mice

We next examined whether brain-specific SIRT1 deletion affects neuronal and behavioral abnormalities in tauP301S mice. SIRT1 deletion accelerated premature mortality in tauP301S mice ( $p=$ 0.0329, log rank; Fig. 3A). Using synaptophysin (SY38) immunoreactivity, we next determined whether SIRT1 deficiency exacerbates tau-mediated synaptic loss. No significant synaptophysin loss was detected in tauP301S mice or in mice lacking SIRT1 alone in the frontal cortex and hippocampus (Fig. 3 B, C). However, brain-specific SIRT1 deletion in tauP301S mice resulted in a significant loss of synaptophysin in the frontal cortex $\left[F_{(3,19)}=\right.$ $7.5, p=0.0079$ (tauP301S; SIRT1 F/F vs Nestin-Cre; tauP301S; SIRT1 F/F), ANOVA $]$ and hippocampus $\left[F_{(3,19)}=11.83, p=\right.$ 0.0019 (tauP301S; SIRT1 F/F vs Nestin-Cre; tauP301S; SIRT1 F/F), ANOVA], indicating that SIRT1 deficiency exacerbated tau-mediated synaptic deficits (Fig. $3 B, C$ ). Interestingly, deletion 
A

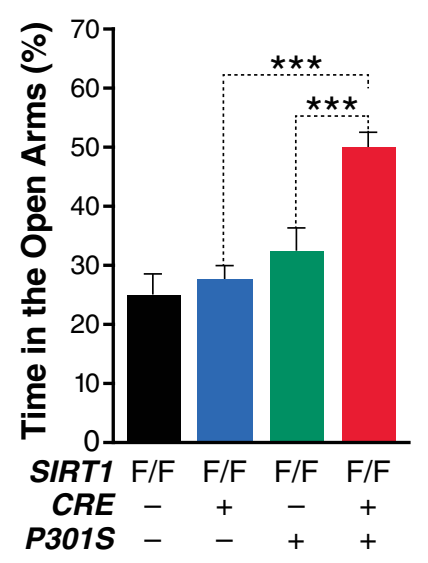

B

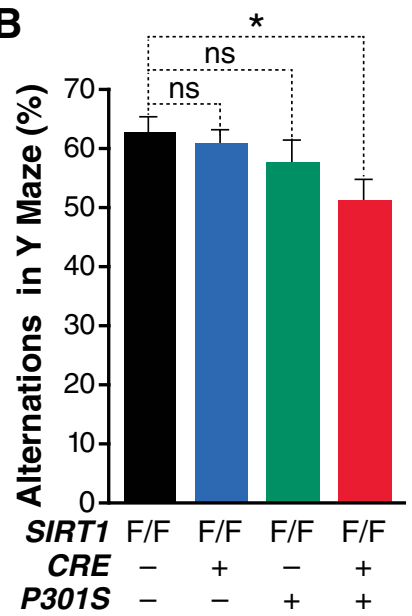

C
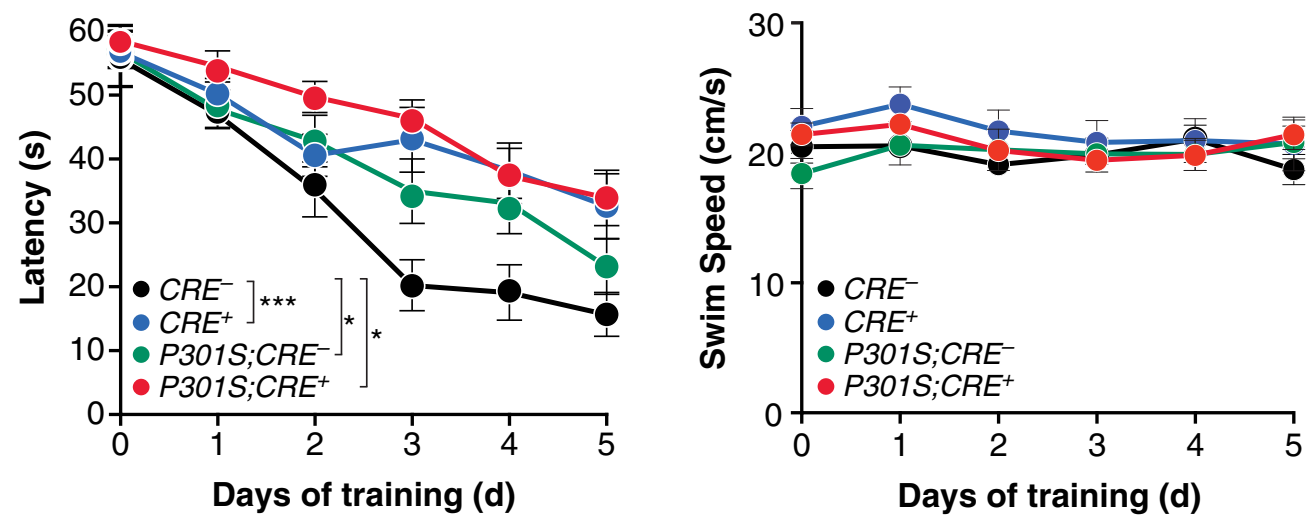

D

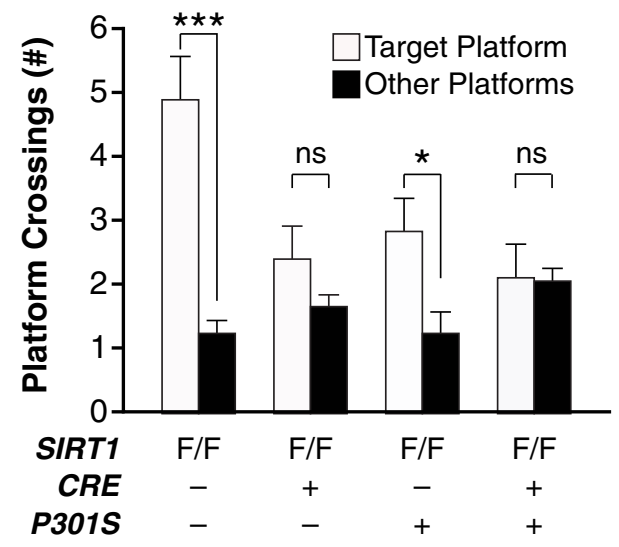

Figure 4. SIRT1 deletion leads to cognitive impairments in tauP301S mice. A, SIRT1 deletion exacerbated tau-mediated behavioral disinhibition in the EPM. P3015 $;$ Cre $^{-}, n=15 ; P 3015^{-}$; $\mathrm{Cre}^{+}, n=15 ; \mathrm{P3O1S}^{+} ; \mathrm{Cre}^{-}, n=14 ; \mathrm{P3O1S}^{+} ; \mathrm{Cre}^{+}, n=10 .^{* * *} p<0.001$, one-way ANOVA, Tukey's post hoc test. $\boldsymbol{B}$, SIRT1 deletion led to tau-mediated spatial memory impairment in the small Y-maze. $n=10-11$ mice per genotype. ${ }^{*} p<0.05$, one-way ANOVA, Dunnett's post hoc test. C, D, SIRT1 deletion impaired spatial learning and memory in the Morris water maze test. $n=10-11$ mice per genotype. C, SIRT1 deletion impaired spatial learning of locating a hidden platform. Swim speed did not differ between the groups. ${ }^{*} p<0.05,{ }^{* * *} p<0.001$, multilevel mixed-effects model. $\boldsymbol{D}$, SIRT1 deletion impaired memory retention in probe trials $24 \mathrm{~h}$ after learning. ${ }^{*} p<0.05 ;{ }^{* *} p<0.001$; paired Student's $t$ test. Values are means \pm SEM.

of SIRT1 did not affect the number of neurofibrillary tangles significantly, as measured with Gallyas silver staining (Fig. 3D).

We next assessed the effects of SIRT1 deficiency on taumediated behavioral deficits. In the EPM test, tauP301S mice deficient in SIRT1, compared with tauP301S mice without the deficiency, spent significantly more time in the open arm $\left[F_{(3,50)}=\right.$ $15.05, p=0.0001$ (tauP301S; SIRT1 F/F vs Nestin-Cre; tauP301S; SIRT1 F/F, ANOVA], suggesting that SIRT1 deletion in tauP301S mice exacerbates behavioral disinhibition (Fig. $4 A$ ). The time spent in the open arm by either tauP301S mice or mice with the SIRT1 deletion alone did not differ significantly from the time spent in the open arm by WT mice (Fig. 4A). In the Y-maze test, tauP301S mice with SIRT1 deletion had significantly fewer spontaneous alterations than WT mice $\left[F_{(3,40)}=2.4, p=0.0405\right.$ (SIRT1 F/F vs Nestin-Cre; tauP301S; SIRT1 F/F), ANOVA], and tauP301S mice or mice with the SIRT1 deletion alone exhibited 
no significant abnormality on spontaneous alterations (Fig. 4B). To examine spatial learning and memory, we used the Morris water maze test. Regardless of human tauP301S expression, SIRT1 deletion led to significantly impaired learning for locating a hidden platform $[p<0.001$ (SIRT1 F/F vs Nestin-Cre; SIRT1 F/F); $p=0.011$ (SIRT1 F/F vs Nestin-Cre; tauP301S; SIRT1 F/F), multilevel mixed effects model; Fig. 4C] and reduced memory retention measured by number of platform crossings (Fig. 4D). Swim speed did not differ between the groups, excluding the possibility that difference in swim speed affected latency for locating a platform (Fig. 4C).

\section{SIRT1 overexpression decreases the} levels of ac-K174 tau and the spread of tau pathology in tauP301S mice We next examined whether SIRT1 overexpression exhibits protective effects against tau pathology in tauP301S mice. Using an AAV vector encoding SIRT1 transgene, we overexpressed SIRT1 in the left hippocampus and injected tau fibrils into the right hippocampus simultaneously to test whether the spread of tau pathology can be ameliorated by SIRT1 overexpression (Fig. 5A). Stereotaxic injections of AAVSIRT1 into the left hippocampus of tauP301S mice successfully induced SIRT1 expression in the left hippocampus, but not in the right hippocampus (Fig. 5B). We first determined whether SIRT1 overexpression decreases the levels of ac-K174 tau. We showed that AAV-SIRT1-induced hippocampal SIRT1 overexpression in tauP301S mice significantly lowered the levels of ac-K174 in the mossy fiber of the left hippocampus, compared with the AAVGFP-injected controls $\left(t_{(21)}=2.5, p=\right.$ 0.0231 , unpaired $t$ test; Fig. $5 C, D)$. The spread of tau pathology was then examined with immunostaining of the MC1 antibody that specifically recognizes pathological conformation of tau (Jicha et al., 1997). In 3-month-old tauP301S mice without injection of tau fibrils, there was no $\mathrm{MC1}$ immunoreactivity in the cell body, but only minimal MC1 staining in mossy fiber of the hippocampus (Fig. $5 E$ ). Injection of tau fibrils into the hippocampus of 2-month-old tauP301S mice resulted in a marked increase of MC1-positive tau pathology in the ipsilateral and contralateral hippocampus of tau fibril injection within 1 month (Fig. 5E). Notably, SIRT1 overexpression significantly reduced the spread of MC1-positive tau pathology to the contralateral hippocampus, compared with the group injected with AAV-GFP ( $p=0.001$, multilevel mixed effects model; Fig. 5E,F). Thus, elevated levels of SIRT1, which lowers ac-K174, suppressed the spread of tau pathology induced by tau fibrils significantly. SEM. Scale bars, $100 \mu \mathrm{m}$.

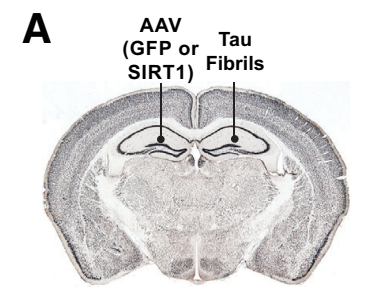

B

C
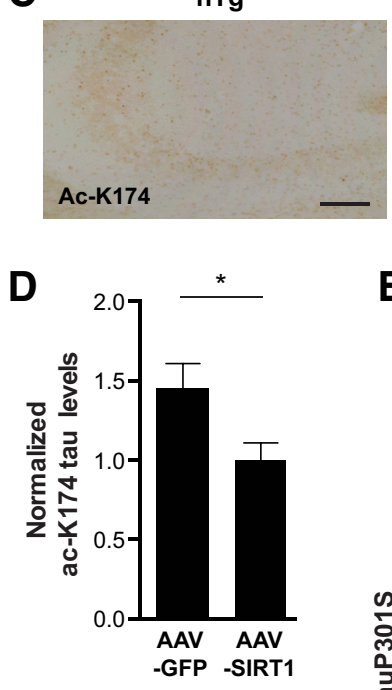

$\mathbf{F}$

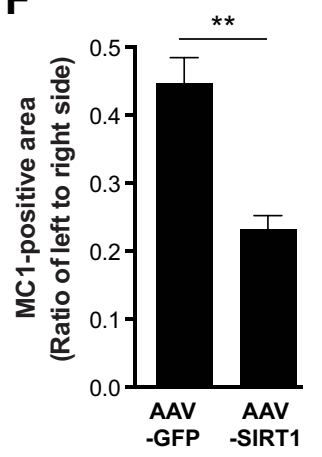

AAV-SIRT1

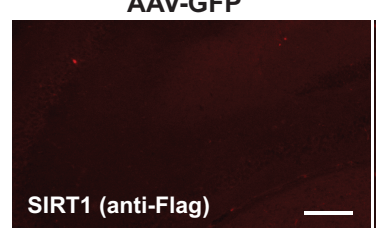

AAV-GFP

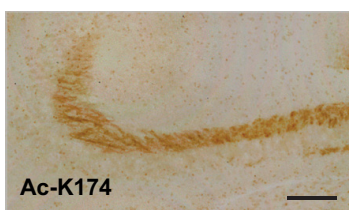

Ac-K174

Figure 5. SIRT1 overexpression decreases the levels of ac-K174 tau and spreading of tau pathology in tauP301S mice. $\boldsymbol{A}$, Schematic diagram of stereotaxic injections of AAV-GFP or AAV-SIRT1 and tau fibrils into the left and right hippocampus, respectively. $B$, Representative images of immunostaining with the flag antibody for visualization of SIRT1 expression in the hippocampus with AAV-GFP and AAV-SIRT1-Flag injections. C, D, Representative images and quantification of immunostaining of ac-K174 tau (AC312) in the mossy fiber of the hippocampus with AAV-GFP and AAV-SIRT1 injections. $n=10-11$ mice per group. ${ }^{*} p<0.05$, unpaired Student's $t$ test. $\boldsymbol{E}$, Representative images of MC1 immunostaining in the hippocampus with AAV-GFP and AAV-SIRT1 injections. Two rows on the top represented negative controls of nontransgenic (nTg) mice and tauP301S mice (Tg) without injection of tau fibrils. $\boldsymbol{F}$, Quantification of MC1-positive area in the left (spread) and right (tau fibrils) sides of the hippocampus with AAV-GFP and AAV-SIRT1 injections. Percentage of MC1-positive area was analyzed, and then the ratio between left and right sides was calculated. $n=35-38$ slices from 10 to 11 mice per group. ${ }^{* *} p<0.01$, multilevel mixed-effects model. Values are means \pm

\section{Discussion}

Our current study provided direct evidence that SIRT1 deacetylates human tau, protects against pathogenic tau spread, and ameliorates synaptic and behavioral deficits in a mouse model of tauopathy. Brain-specific SIRT1 deletion elevated levels of acK174 tau in brains of tauP301S mice. Premature death, synaptic loss, and behavioral disinhibition were exacerbated by deletion of SIRT1 in the brains of tauP301S transgenic mice. By contrast, increasing SIRT1 with AAV injection into the hippocampus re- 
duced the levels of ac-K174 tau and ameliorated the spread of pathogenic tau in tauP301S transgenic mice.

SIRT1 and HDAC6 have abilities to deacetylate tau (Min et al., 2010; Cook et al., 2014; Tsushima et al., 2015). Using quantitative proteomic analyses, we showed directly that SIRT1 removes acetyl groups from five acetyllysine residues of tau in cultured cells. Further analyses of mouse brains were performed using specific antibodies AC312 and MAB359, targeting two of those acetyllysine residues (i.e., K174 and K274), whose acetylation was elevated in tauopathy brains (Grinberg et al., 2013; Min et al., 2015; Sohn et al., 2016; Tracy et al., 2016). Interestingly, we found that, in tauP301S mouse brains, SIRT1 deacetylates human tau preferentially at K174 without affecting the acetylation status at K274 significantly. Other acetylated lysines, such as the lysines in KXGS motifs, appear to be deacetylated by HDAC6 (Cook et al., 2014). The exact mechanisms for the preferential deacetylation are unknown. Different ac-tau species might have different subcellular localizations, where SIRT1 may or may not have access.

Loss of SIRT1 may contribute to tau hyperacetylation in tauopathy brains. While levels of pathogenic ac-K174 tau are increased in AD brains (Min et al., 2015), mRNA and protein levels of SIRT1 are reduced and inversely correlated with tau accumulation in $\mathrm{AD}$ brains (Julien et al., 2009). Lowering SIRT1 in primary neurons increases tau acetylation at $\mathrm{N}$-terminal lysines, including K174 (Min et al., 2010). Pharmacological inhibition of HDAC6 increases lysine acetylation in KXGS motifs of tau (Cook et al., 2014), and autoacetyltransferase activity of tau modulates lysine acetylation mainly in the microtubule-binding domain (Cohen et al., 2013, 2016). Thus, different acetyltransferases and deacetylases could be involved in modulating specific acetylation at different lysine residues.

Different from mice expressing K174Q tau, in which every human tau molecule is pseudoacetylated at K174 (Min et al., 2015), brain-specific SIRT1 deletion elevated ac-K174 on a subset of tau. Interestingly, SIRT1 deletion increased total tau levels specifically in the postsynaptic fractions, suggesting the specific roles of ac-tau in regulating tau homeostasis at subcellular levels. Notably, the elevation of total tau and ac-tau induced by brainspecific SIRT1 is accompanied by reduced synaptophysin immunoreactivity in tauP301S transgenic mice. The mechanism by which SIRT1 deletion results in disruption of synapses in a tauopathy mouse model remains to be established, but increased tau levels in the dendritic spine could lead to disruption of the postsynaptic spines (Zempel et al., 2010, 2013) and concomitant loss of presynaptic boutons due to lack of stabilizing interaction at the synapse (Lin and Koleske, 2010). Postsynaptic increase of tau acetylation at K174 could contribute to increased levels of tau in the postsynaptic compartment since ac-K174 tau mimic slows down its degradation (Min et al., 2015). In addition, since the phosphorylation state of tau regulates postsynaptic invasion of tau (Hoover et al., 2010; Zempel et al., 2010), increased phosphorylation at S202/T205 by K174 acetylation (Min et al., 2015) could further facilitate postsynaptic mislocalization of tau. On the other hand, we cannot exclude the possibility that SIRT1 deletion leads to synapse loss in tauP301S transgenic mice via an ac-tau-independent mechanism, especially in their old ages. SIRT1 deletion can lead to decreased BNDF expression (Gao et al., 2010; Zocchi and Sassone-Corsi, 2012) and downregulated BDNF signaling may increase vulnerability to synapse loss (Lin and Koleske, 2010).

We demonstrated exacerbation of tau-mediated mortality and behavioral disinhibition by SIRT1 deficiency in a mouse model of FTDP-17. Accumulating studies on SIRT1 have docu- mented the neuroprotective and prosurvival roles of SIRT1 in neurodegenerative diseases (Gan and Mucke, 2008; Min et al., 2013; Ng et al., 2015). SIRT1 deficiency leads to impaired memory and spatial learning in physiological conditions (Gao et al., 2010; Michán et al., 2010). However, inhibition of SIRT1 alleviates pathology and restores cognition in pathological mouse models of Huntington's disease (Smith et al., 2014) and AD (Green et al., 2008), respectively. These findings highlight the complexity of downstream signaling induced by SIRT1. Since the tau tangle load was not altered by SIRT1 deficiency in tauP301S mice, cellular dysfunction induced by soluble tau may underlie behavioral deficits and worsening of mortality in SIRT1-deleted tauP301S mice. Given the pleotropic role of SIRT1 in regulation neuronal function (Gan and Mucke, 2008; Ng et al., 2015), it is possible that other factors in addition to acetylated tau could contribute to the exacerbation of mortality and behavioral disinhibition in SIRT1-deleted tauP301S mice.

AAV-mediated SIRT1 overexpression in the hippocampus decreased levels of ac-K174 tau and attenuated spreading of tau pathology in tauP301S mice. The transcellular spread of tau pathology has been used to stage the progression of tauopathies, including $\mathrm{AD}$ (Braak and Braak, 1991). Inoculation of brain extracts or tau fibrils into mouse brains induces seeding and spreading of tau pathology from the site of injection to anatomically connected brain regions (Clavaguera et al., 2009; Iba et al., 2013). We observed MC1-positive tau inclusions in the contralateral hippocampus within 4 weeks after injection of tau fibrils, which is consistent with the previous study on spreading of tau pathology in tauP301S mice (Iba et al., 2013). Whether reduction of ac-K174 tau in recipient neurons contributes to the attenuated spread of pathological tau remains to be investigated. Since SIRT1 can accelerate rates of autophagy by deacetylating proteins essential for autophagy (Lee et al., 2008), SIRT1 overexpression in recipient neurons may promote removal of internalized pathological tau via increased autophagy (Wang and Mandelkow, 2012), leading to fewer MC1-positive tau inclusions in the recipient neurons. In addition, the propagation of tau pathology may result from the progression of neuroinflammation via microglia (Maphis et al., 2015). Since SIRT1 deficiency elevates levels of proinflammatory cytokine (Cho et al., 2015), SIRT1 overexpression may attenuate the spreading of tau pathology by reducing neuroinflammation. Thus, it will be interesting to determine how SIRT1 modulates propagation of tau pathology.

In summary, our results showed a protective role of SIRT1 in regulating disease progression of tauopathy in the brain. SIRT1 deficiency in the brain elevates ac-tau and leads to synapse loss and behavioral deficits, whereas SIRT1 overexpression reduces ac-tau and ameliorates propagation of tau pathology. These studies support SIRT1 activation as a potential therapeutic strategy for $\mathrm{AD}$ and related tauopathies.

\section{References}

Barghorn S, Biernat J, Mandelkow E (2005) Purification of recombinant tau protein and preparation of Alzheimer-paired helical filaments in vitro. Methods Mol Biol 299:35-51. Medline

Braak H, Braak E (1991) Neuropathological stageing of Alzheimer-related changes. Acta Neuropathol 82:239-259. CrossRef Medline

Brettschneider J, Del Tredici K, Lee VM, Trojanowski JQ (2015) Spreading of pathology in neurodegenerative diseases: a focus on human studies. Nat Rev Neurosci 16:109-120. CrossRef Medline

Cho SH, Chen JA, Sayed F, Ward ME, Gao F, Nguyen TA, Krabbe G, Sohn PD, Lo I, Minami S, Devidze N, Zhou Y, Coppola G, Gan L (2015) SIRT1 deficiency in microglia contributes to cognitive decline in aging and neurodegeneration via epigenetic regulation of IL-1 $\beta$. J Neurosci 35:807818. CrossRef Medline 
Clavaguera F, Bolmont T, Crowther RA, Abramowski D, Frank S, Probst A, Fraser G, Stalder AK, Beibel M, Staufenbiel M, Jucker M, Goedert M, Tolnay M (2009) Transmission and spreading of tauopathy in transgenic mouse brain. Nat Cell Biol 11:909-913. CrossRef Medline

Cohen TJ, Guo JL, Hurtado DE, Kwong LK, Mills IP, Trojanowski JQ, Lee VM (2011) The acetylation of tau inhibits its function and promotes pathological tau aggregation. Nat Commun 2:252. CrossRef Medline

Cohen TJ, Friedmann D, Hwang AW, Marmorstein R, Lee VM (2013) The microtubule-associated tau protein has intrinsic acetyltransferase activity. Nat Struct Mol Biol 20:756-762. CrossRef Medline

Cohen TJ, Constance BH, Hwang AW, James M, Yuan CX (2016) Intrinsic tau acetylation is coupled to auto-proteolytic tau fragmentation. PLoS One 11:e0158470. CrossRef Medline

Cook C, Carlomagno Y, Gendron TF, Dunmore J, Scheffel K, Stetler C, Davis M, Dickson D, Jarpe M, DeTure M, Petrucelli L (2014) Acetylation of the KXGS motifs in tau is a critical determinant in modulation of tau aggregation and clearance. Hum Mol Genet 23:104-116. CrossRef Medline

Cox J, Mann M (2008) MaxQuant enables high peptide identification rates, individualized p.p.b.-range mass accuracies and proteome-wide protein quantification. Nat Biotechnol 26:1367-1372. CrossRef Medline

Gan L, Mucke L (2008) Paths of convergence: sirtuins in aging and neurodegeneration. Neuron 58:10-14. CrossRef Medline

Gao J, Wang WY, Mao YW, Gräff J, Guan JS, Pan L, Mak G, Kim D, Su SC, Tsai LH (2010) A novel pathway regulates memory and plasticity via SIRT1 and miR-134. Nature 466:1105-1109. CrossRef Medline

Green KN, Steffan JS, Martinez-Coria H, Sun X, Schreiber SS, Thompson LM, LaFerla FM (2008) Nicotinamide restores cognition in Alzheimer's disease transgenic mice via a mechanism involving sirtuin inhibition and selective reduction of Thr231-phosphotau. J Neurosci 28:11500-11510. CrossRef Medline

Grinberg LT, Wang X, Wang C, Sohn PD, Theofilas P, Sidhu M, Arevalo JB, Heinsen H, Huang EJ, Rosen H, Miller BL, Gan L, Seeley WW (2013) Argyrophilic grain disease differs from other tauopathies by lacking tau acetylation. Acta Neuropathol 125:581-593. CrossRef Medline

Hellman U, Wernstedt C, Góñez J, Heldin CH (1995) Improvement of an "In-Gel" digestion procedure for the micropreparation of internal protein fragments for amino acid sequencing. Anal Biochem 224:451-455. CrossRef Medline

Hoover BR, Reed MN, Su J, Penrod RD, Kotilinek LA, Grant MK, Pitstick R, Carlson GA, Lanier LM, Yuan LL, Ashe KH, Liao D (2010) Tau mislocalization to dendritic spines mediates synaptic dysfunction independently of neurodegeneration. Neuron 68:1067-1081. CrossRef Medline

Iba M, Guo JL, McBride JD, Zhang B, Trojanowski JQ, Lee VM (2013) Synthetic tau fibrils mediate transmission of neurofibrillary tangles in a transgenic mouse model of Alzheimer's-like tauopathy. J Neurosci 33: 1024-1037. CrossRef Medline

Irwin DJ, Cohen TJ, Grossman M, Arnold SE, McCarty-Wood E, Van Deerlin VM, Lee VM, Trojanowski JQ (2013) Acetylated tau neuropathology in sporadic and hereditary tauopathies. Am J Pathol 183:344-351. CrossRef Medline

Jicha GA, Bowser R, Kazam IG, Davies P (1997) Alz-50 and MC-1, a new monoclonal antibody raised to paired helical filaments, recognize conformational epitopes on recombinant tau. J Neurosci Res 48:128-132. CrossRef Medline

Julien C, Tremblay C, Emond V, Lebbadi M, Salem N Jr, Bennett DA, Calon F (2009) Sirtuin 1 reduction parallels the accumulation of tau in Alzheimer disease. J Neuropathol Exp Neurol 68:48 -58. CrossRef Medline

Kim D, Nguyen MD, Dobbin MM, Fischer A, Sananbenesi F, Rodgers JT, Delalle I, Baur JA, Sui G, Armour SM, Puigserver P, Sinclair DA, Tsai LH (2007) SIRT1 deacetylase protects against neurodegeneration in models for Alzheimer's disease and amyotrophic lateral sclerosis. EMBO J 26: 3169-3179. CrossRef Medline

Lee IH, Cao L, Mostoslavsky R, Lombard DB, Liu J, Bruns NE, Tsokos M, Alt FW, Finkel T (2008) A role for the NAD-dependent deacetylase Sirtl in the regulation of autophagy. Proc Natl Acad Sci U S A 105:3374-3379. CrossRef Medline

Lin YC, Koleske AJ (2010) Mechanisms of synapse and dendrite maintenance and their disruption in psychiatric and neurodegenerative disorders. Annu Rev Neurosci 33:349-378. CrossRef Medline

Maphis N, Xu G, Kokiko-Cochran ON, Jiang S, Cardona A, Ransohoff RM, Lamb BT, Bhaskar K (2015) Reactive microglia drive tau pathology and contribute to the spreading of pathological tau in the brain. Brain 138: 1738-1755. CrossRef Medline

Michán S, Li Y, Chou MM, Parrella E, Ge H, Long JM, Allard JS, Lewis K, Miller M, Xu W, Mervis RF, Chen J, Guerin KI, Smith LE, McBurney MW, Sinclair DA, Baudry M, de Cabo R, Longo VD (2010) SIRT1 is essential for normal cognitive function and synaptic plasticity. J Neurosci 30:9695-9707. CrossRef Medline

Min SW, Cho SH, Zhou Y, Schroeder S, Haroutunian V, Seeley WW, Huang EJ, Shen Y, Masliah E, Mukherjee C, Meyers D, Cole PA, Ott M, Gan L (2010) Acetylation of tau inhibits its degradation and contributes to tauopathy. Neuron 67:953-966. CrossRef Medline

Min SW, Sohn PD, Cho SH, Swanson RA, Gan L (2013) Sirtuins in neurodegenerative diseases: an update on potential mechanisms. Front Aging Neurosci 5:53. CrossRef Medline

Min SW, Chen X, Tracy TE, Li Y, Zhou Y, Wang C, Shirakawa K, Minami SS, Defensor E, Mok SA, Sohn PD, Schilling B, Cong X, Ellerby L, Gibson BW, Johnson J, Krogan N, Shamloo M, Gestwicki J, Masliah E, et al. (2015) Critical role of acetylation in tau-mediated neurodegeneration and cognitive deficits. Nat Med 21:1154-1162. CrossRef Medline

Morris M, Knudsen GM, Maeda S, Trinidad JC, Ioanoviciu A, Burlingame AL, Mucke L (2015) Tau post-translational modifications in wild-type and human amyloid precursor protein transgenic mice. Nat Neurosci 18:1183-1189. CrossRef Medline

Ng F, Wijaya L, Tang BL (2015) SIRT1 in the brain-connections with agingassociated disorders and lifespan. Front Cell Neurosci 9:64. CrossRef Medline

Qin W, Yang T, Ho L, Zhao Z, Wang J, Chen L, Zhao W, Thiyagarajan M, MacGrogan D, Rodgers JT, Puigserver P, Sadoshima J, Deng H, Pedrini S, Gandy S, Sauve AA, Pasinetti GM (2006) Neuronal SIRT1 activation as a novel mechanism underlying the prevention of Alzheimer disease amyloid neuropathology by calorie restriction. J Biol Chem 281:21745-21754. CrossRef Medline

Rockenstein E, Overk CR, Ubhi K, Mante M, Patrick C, Adame A, Bisquert A, Trejo-Morales M, Spencer B, Masliah E (2015) A novel triple repeat mutant tau transgenic model that mimics aspects of Pick's disease and fronto-temporal tauopathies. PLoS One 10:e0121570. CrossRef Medline

Rosenfeld J, Capdevielle J, Guillemot JC, Ferrara P (1992) In-gel digestion of proteins for internal sequence analysis after one- or two-dimensional gel electrophoresis. Anal Biochem 203:173-179. CrossRef Medline

Smith MR, Syed A, Lukacsovich T, Purcell J, Barbaro BA, Worthge SA, Wei SR, Pollio G, Magnoni L, Scali C, Massai L, Franceschini D, Camarri M, Gianfriddo M, Diodato E, Thomas R, Gokce O, Tabrizi SJ, Caricasole A, Landwehrmeyer B, et al. (2014) A potent and selective sirtuin 1 inhibitor alleviates pathology in multiple animal and cell models of Huntington's disease. Hum Mol Genet 23:2995-3007. CrossRef Medline

Sohn PD, Tracy TE, Son HI, Zhou Y, Leite RE, Miller BL, Seeley WW, Grinberg LT, Gan L (2016) Acetylated tau destabilizes the cytoskeleton in the axon initial segment and is mislocalized to the somatodendritic compartment. Mol Neurodegener 11:47. CrossRef Medline

Tracy TE, Sohn PD, Minami SS, Wang C, Min SW, Li Y, Zhou Y, Le D, Lo I, Ponnusamy R, Cong X, Schilling B, Ellerby LM, Huganir RL, Gan L (2016) Acetylated tau obstructs KIBRA-mediated signaling in synaptic plasticity and promotes tauopathy-related memory loss. Neuron 90:245260. CrossRef Medline

Tsushima H, Emanuele M, Polenghi A, Esposito A, Vassalli M, Barberis A, Difato F, Chieregatti E (2015) HDAC6 and RhoA are novel players in abeta-driven disruption of neuronal polarity. Nat Commun 6:7781. CrossRef Medline

Wang Y, Mandelkow E (2012) Degradation of tau protein by autophagy and proteasomal pathways. Biochem Soc Trans 40:644-652. CrossRef Medline

Wang Y, Mandelkow E (2016) Tau in physiology and pathology. Nat Rev Neurosci 17:5-21. CrossRef Medline

Zempel H, Thies E, Mandelkow E, Mandelkow EM (2010) A $\beta$ oligomers cause localized $\mathrm{Ca}^{2+}$ elevation, missorting of endogenous tau into dendrites, tau phosphorylation, and destruction of microtubules and spines. J Neurosci 30:11938-11950. CrossRef Medline

Zempel H, Luedtke J, Kumar Y, Biernat J, Dawson H, Mandelkow E, Mandelkow EM (2013) Amyloid-beta oligomers induce synaptic damage via tau-dependent microtubule severing by TTLL6 and spastin. EMBO J 32: 2920-2937. CrossRef Medline

Zocchi L, Sassone-Corsi P (2012) SIRT1-mediated deacetylation of MeCP2 contributes to BDNF expression. Epigenetics 7:695-700. CrossRef Medline 